\title{
solid

\section{Quantum entanglement and order parameter in a paired finite Fermi system}

\author{
Zafer Gedik* \\ Department of Physics, Bilkent University, Bilkent, Ankara 06533, Turkey
}

Received 23 July 2002; received in revised form 15 August 2002; accepted 16 August 2002 by M. Cardona

\begin{abstract}
We study the pairing correlations in a finite Fermi system from the quantum entanglement point of view. We investigate the relation between the order parameter, which has been introduced recently to describe both finite and infinite superconductors, and the concurrence. For a proper definition of the concurrence, we argue that a possible generalization of the spin flip transformation is a time reversal operation. While for a system with indefinite number of particles concurrence is a good measure of entanglement, for a finite system it does not distinguish between normal and superconducting states. We propose that the expectation value of the radial operator for the total pseudospin can be used to identify entanglement of pairing.
\end{abstract} (C) 2002 Elsevier Science Ltd. All rights reserved.

PACS: 03.65.-w; 03.67.Lx; 89.70. + c; 74.20.Fg; 74.20.-z

Keywords: A. Finite Fermi systems; D. Order parameter; D. Quantum entanglement

Entanglement is a fundamental quantum mechanical property [1] which plays a central role in the quantum information theory [2]. On the other hand, proposed measures of entanglement, including the entanglement of formation which quantifies the resources needed to create a given entangled state [3], are generally not very proper for analytical calculations. Making use of a spin flip transformation, Wootters [4] introduced the so-called the concurrence to simplify the notion of entanglement of formation and Martín-Delgado [5] extended and applied his results to a many-body problem, namely the BCS ground state of superconductivity $[6,7]$. In this work, we examine the same problem for a finite system where the number of fermions is fixed. For this purpose, we make use of an order parameter proposed recently to describe both microcanonical and grandcanonical superconductors [8].

Experimental works on superconducting metallic islands at nanometer scale raised questions about pairing corre-

\footnotetext{
* Address (after September 2002): Faculty of Engineering and Natural Sciences, Sabanci University, 34956 Tuzla, Istanbul, Turkey. Tel.: +90-216-483-9610; fax: +90-216-483-9550.

E-mail address: gedik@sabanciuniv.edu (Z. Gedik).
}

lations [9-13]. For a bulk system, superconductivity can be described by a complex order parameter $\Delta$. The equations have the symmetry that if $\Delta$ is a solution, then $\mathrm{e}^{\mathrm{i} \theta} \Delta$ is also a solution [6,7]. However, in a finite system with fixed number of electrons, the order parameter $\Delta=\left\langle c_{-\mathbf{k} \downarrow} c_{\mathbf{k} \uparrow}\right\rangle$ vanishes since the operator does not conserve the number of fermions. Here, $c_{-\mathbf{k} \downarrow}$ and $c_{\mathbf{k} \uparrow}$ are the annihilation operators for time reversed states $|-\mathbf{k} \downarrow\rangle$ and $|\mathbf{k} \uparrow\rangle$, respectively. In this case, superconductivity can be identified by nonvanishing number parity effect parameter $\Delta_{\mathrm{P}}$ since the ground state energy of the system increases or decreases, depending upon whether the total number becomes odd or even, by addition of a new electron [14,15]. Recently, an order parameter has been proposed to unify the order parameter $\Delta$ of the bulk limit and the number parity effect parameter $\Delta_{\mathrm{P}}$ of the nanoscopic superconductors [8]. Using the pseudospin representation $[16,17]$ and the $S U(2)$ phase states [18] a quantum phase has been defined for a superconductor with discrete energy levels along with modulus of the order parameter which becomes equal to $\Delta_{\mathrm{p}}$. As we go from the nanoscopic limit to the bulk superconductor it has been shown that the number parity effect parameter and the SU(2) phase go to the amplitude and the phase of the bulk order 
parameter, respectively. On the other hand, we can think of the long-range order in the superconducting phase as an entangled state of Cooper pairs. In this paper, we first discuss how to calculate concurrence as a measure of entanglement and we examine the relation between entanglement and the order parameter. We show that while for a system with indefinite number of fermions concurrence is a good measure of entanglement, for a finite system it does not identify the pairing correlations. As a possible solution, we propose the amplitude of our order parameter, which is nothing but the expectation value of the radial operator of the total pseudospin, to detect entanglement of Cooper pairs.

For a finite Fermi system, such as a nanoscopic superconductor, energy levels are also finite and discrete and hence we can use a reduced form of the BCS model [19] which was applied in nuclear physics and which has an exact solution [20]. The model Hamiltonian is

$H=\sum_{j, \sigma} \epsilon_{j} c_{j \sigma}^{\dagger} c_{j \sigma}-g \sum_{j, j^{\prime}} c_{j \uparrow}^{\dagger} c_{j \downarrow}^{\dagger} c_{j^{\prime} \downarrow} c_{j^{\prime} \uparrow}$,

where $g$ is the pairing coupling constant for the timereversed states $|j \uparrow\rangle$ and $|j \downarrow\rangle$, both having the energy $\epsilon_{j}$. Here, $c_{j \sigma}^{\dagger}\left(c_{j \sigma}\right)$ is the creation (annihilation) operator for state $|j \sigma\rangle$ where $j \in\{1, \ldots, \Omega\}$ and $\sigma \in\{\uparrow, \downarrow\}$. For the model Hamiltonian introduced above it has been shown that there exists a number parity effect, namely the ground state energy for even number of electrons is lower in comparison to neighboring odd number states [21-24] including degenerate case [25].

The key point in Wootters' formulation of concurrence is the spin flip transformation. For a pure state of a single qubit $|\psi\rangle$ it is defined by

$|\tilde{\psi}\rangle=\sigma_{y}\left|\psi^{*}\right\rangle$,

where $\left|\psi^{*}\right\rangle$ is obtained from $|\psi\rangle$ by taking complex conjugates of expansion coefficients and $\sigma_{y}$ is Pauli spin matrix. For a single spin- $1 / 2$ particle, this is nothing but the time reversal operation. The spin degree of freedom that we discuss here should not be mixed with the pseudospin to be introduced below. For the many body case, a natural extension of the spin flip operation is the time reversal operation [5]. The action of the time reversal operator $U_{\mathrm{T}}$ on the creation operator is:

$U_{\mathrm{T}} c_{j \sigma}^{\dagger} U_{\mathrm{T}}^{\dagger}=c_{j-\sigma}^{\dagger}$.

A similar relation holds for the annihilation operator. To find the transformed state $|\tilde{\psi}\rangle$ (in the active picture) we can simply rewrite the transformed Hamiltonian (in the passive picture) and evaluate the corresponding eigenstate.

To define the order parameter, we introduce the pseudospin variables [16,17]

$s_{j}^{z}=\frac{1}{2}\left(c_{j \uparrow}^{\dagger} c_{j \uparrow}+c_{j \downarrow}^{\dagger} c_{j \downarrow}-1\right), \quad s_{j}^{-}=c_{j \downarrow} c_{j \uparrow}=\left(s_{j}^{+}\right)^{\dagger}$,

which obey the fundamental commutation relations of the
SU(2) algebra:

$\left[s_{i}^{+}, s_{j}^{-}\right]=2 \delta_{i j} s_{j}^{z}, \quad\left[s_{i}^{z}, s_{j}^{ \pm}\right]= \pm \delta_{i j} s_{j}^{ \pm}$.

It is possible to rewrite the model Hamiltonian as:

$H=\sum_{j} 2 \epsilon_{j}\left(s_{j}^{z}+\frac{1}{2}\right)-g \sum_{i j} s_{i}^{+} s_{j}^{-}$.

The mapping from the Fermi operators to the pseudospin operators is possible as long as all single particle states are doubly occupied. Since the original Hamiltonian (1) contains no terms which couple a singly occupied level to others, the only role of such states will be blocking from pairing interaction. Therefore, the summations in Eq. (6) are over doubly occupied and empty states only. Both the above mapping and the BCS wave function [6] lack proper antisymmetrization due to separate treatment of singly occupied states, but since the model Hamiltonian (1) does not involve any scatterings into or out of such states, antisymmetrization with respect to interlevel pair exchange and intrapair electron exchange is sufficient.

Given SU(2) algebra, for example the one generated by the components of the total pseudospin operator $\mathbf{s}=\sum_{i} \mathbf{s}_{i}$, we can introduce [18] the radial operator defined by

$s_{\mathrm{r}}=\sqrt{s^{+} s^{-}}$,

and the exponential of the phase operator given by:

$E=\sum_{m=-s}^{m=s}|S ; s m+1\rangle\langle S ; s m|$.

Here, $|S ; s m\rangle$ are simultaneous eigenstates of $\mathbf{s}^{2}$ and $s_{z}$ operators with eigenvalues $s(s+1)$ and $m$, respectively. The label $S$ has been introduced to distinguish them from the phase states to be defined below. For integer $s$ or in the so called Bose sector, the eigenstate of $E$ with eigenvalue $\exp (-\mathrm{i} 2 \pi n /(2 s+1))$ is evaluated to be

$|\theta ; s n\rangle=\frac{1}{\sqrt{2 s+1}} \sum_{m=-s}^{m=s} \exp \left[\mathrm{i} \frac{2 \pi n}{2 s+1} m\right]|S ; s m\rangle$,

and a similar expression holds for half integer $s$ or in the Fermi sector.

In terms of the radial and the exponential of the phase operators for the total pseudospin, it is possible to rewrite the interaction part of the Hamiltonian (6) as $-g s_{\mathrm{r}} E E^{\dagger} s_{\mathrm{r}}$. Since $E$ is unitary, we have $E E^{\dagger}=I$ but we are going to keep $E$ and $E^{\dagger}$ without cancellation to introduce the phase properly. Now, we define $\left\langle s_{\mathrm{r}}\right\rangle$ and $\langle E\rangle$ as the amplitude and phase of the order parameter, respectively. It has been proven that $\left\langle s_{\mathrm{r}}\right\rangle$ becomes identical to the modulus of the BCS order parameter in the bulk limit while in the nanoscopic limit it reduces to the number parity effect parameter $\Delta_{\mathrm{P}}$ [8]. Furthermore, in the bulk limit, $\langle E\rangle$ becomes identical to the phase of the BCS order parameter. Next, we examine how the amplitude and the phase of the order parameter is transformed under the time reversal operation $T$. Eq. (3) implies that the components of the total 
pseudospin operator $\mathbf{s}$ transform according to:

$U_{\mathrm{T}} s_{x} U_{\mathrm{T}}^{\dagger}=-s_{x}, \quad U_{\mathrm{T}} s_{y} U_{\mathrm{T}}^{\dagger}=-s_{y}$,

$U_{\mathrm{T}} s_{z} U_{\mathrm{T}}^{\dagger}=s_{z}$.

The transformation has immediate consequences on the order parameter. First, the amplitude $\left\langle s_{\mathrm{r}}\right\rangle$ remains unchanged. Second, since $\langle E\rangle=\left\langle s^{+}\right\rangle /\left\langle\mathbf{s}^{2}-s_{z}^{2}-s_{z}\right\rangle$, the exponential of the phase expectation value acquires a minus sign or phase angle change by $\pi$. This is consistent with the prediction of BCS mean field treatment [5]. Here, we note that pseudospin operator does not transform exactly like spin operator $\sigma$ whose components satisfy

$U_{\mathrm{T}} \sigma_{x} U_{\mathrm{T}}^{\dagger}=-\sigma_{x}, \quad U_{\mathrm{T}} \sigma_{y} U_{\mathrm{T}}^{\dagger}=\sigma_{y}$,

$U_{\mathrm{T}} \sigma_{z} U_{\mathrm{T}}^{\dagger}=-\sigma_{z}$

in the standard representation of Pauli matrices [26].

For a given state $|\psi\rangle$, the central quantity concurrence is defined by [4]:

$C(\psi)=|\langle\psi \mid \tilde{\psi}\rangle|$.

Since $\left[s_{\mathrm{r}}, s_{z}\right]=0, s_{\mathrm{r}}$ leads to a good quantum number even for a finite system. The eigenstates and including the ground state of the model Hamiltonian will be of the form

$\left|\psi_{m}\right\rangle=\sum_{s} c_{s}|S ; s m\rangle$

because the interaction term commutes with $\mathbf{s}^{2}$ and $s_{z}$ while the single particle part commutes with the latter, only. In general, the total spin in is multiply degenerate. We can calculate the expectation value of the radial operator as:

$\left\langle s_{\mathrm{r}}\right\rangle=\sum_{s}\left|c_{s}\right|^{2} \sqrt{s(s+1)-m(m-1)}$.

Since the problem is exactly solvable, $c_{s}$ coefficients can be found numerically [27]. In terms of these coefficients we can write down the transformed state which will be same as Eq. (13) except that all coefficients will be replaced by their complex conjugates. Then we evaluate the concurrence as:

$C(\psi)=\left|\sum_{s} c_{s}^{2}\right|$.

The BCS ground state, which is a superposition of states of the form Eq. (13) with different $m$ values, corresponds to phase states $|\theta ; s n\rangle$ in our notation [8]. In other words, it is an extended state in $m$-space and $C(\mathrm{BCS})$ can be calculated explicitly [5]. Here, we can evaluate the same quantity for the phase states. However, since the phase states are defined for a given $s$ value, we need to generalize Eq. (13) by

$\left|\psi_{\theta}\right\rangle \propto \sum_{m} \mathrm{e}^{\mathrm{i} m \theta}\left|\psi_{m}\right\rangle$, from which we find that:

$C\left(\psi_{\theta}\right) \propto\left|\sum_{s} c_{s}^{2}\right|\left|\sum_{m} \mathrm{e}^{\mathrm{i} 2 m \theta}\right|$.

The second term implies that concurrence vanishes in contrast to the Fermi sea state. Hence, concurrence is a distinguishing parameter for entanglement of Cooper pairs.

For a state with real expansion coefficients, assuming Eq. (13) is normalized, concurrence is unity, i.e. it is same as unpaired state. This is the case for a system with fixed number of fermions. A simple and analytically solvable example is a system composed of a single, $d$-fold degenerate energy level $[28,29]$. Therefore, microcanonical entanglement of pairing, which we define by following MartínDelgado as the difference between concurrence values of the Fermi sea and the BCS ground state, vanishes. Although concurrence does not distinguish between the normal and the superconducting states, amplitude of the order parameter $\left\langle s_{\mathrm{r}}\right\rangle$ still identifies pairing correlations and hence it can be used as a signature of entanglement of Cooper pairs.

In conclusion, for a superconducting system with indefinite number of particles, concurrence vanishes while it is unity at the Fermi sea and hence it can be utilized to detect the existence of entanglement. On the other hand, for a finite system it is unity in the superconducting state, too. However, the order parameter of superconductivity which we propose can still be used to identify entanglement of Cooper pairs.

\section{Acknowledgements}

It is my pleasure to be a contributor in the Festschrift in the honor of my ex-supervisor and colleague Prof. Salim Ciraci's 60th birthday. I acknowledge the hospitality of NIST Center for Neutron Research at Gaithersburg where part of the work was performed. I also thank to O. Gülseren and T. Yildirım for helpful discussions. This work was partially supported by the Turkish Academy of Sciences TÜBA/GEBİP Program.

\section{References}

[1] E. Schrödinger, Proc. Cambridge Phil. Soc. 31 (1935) 555.

[2] D. Bouwmeester, A. Ekert, A. Zeilinger (Eds.), The Physics of Quantum Information, Springer, Berlin, 2000.

[3] C.H. Bennett, D.P. DiVincenzo, J. Smolin, W.K. Wootters, Phys. Rev. A 54 (1996) 3824.

[4] W.K. Wootters, Phys. Rev. Lett. 80 (1998) 2245.

[5] M.A. Martín-Delgado, quant-ph/0207026.

[6] J. Bardeen, L.N. Cooper, J.R. Schrieffer, Phys. Rev. 108 (1957) 1175.

[7] N.N. Bogolubov, Sov. Phys. JETP 7 (1958) 41.

[8] Z. Gedik, Phys. Rev. B, submitted. 
[9] M.T. Tuominen, J.M. Hergenrother, T.S. Tighe, M. Tinkham, Phys. Rev. Lett. 69 (1992) 1997.

[10] P. Lafarge, P. Joyez, D. Esteve, C. Urbina, M.H. Devoret, Phys. Rev. Lett. 70 (1993) 994.

[11] D.C. Ralph, C.T. Black, M. Tinkham, Phys. Rev. Lett. 74 (1995) 3241.

[12] D.C. Ralph, C.T. Black, M. Tinkham, Phys. Rev. Lett. 76 (1996) 688.

[13] D.C. Ralph, C.T. Black, M. Tinkham, Phys. Rev. Lett. 78 (1997) 4087.

[14] K.A. Matveev, A.I. Larkin, Phys. Rev. Lett. 78 (1997) 3749.

[15] H. Flocard, in: C. Guet, P. Hobza, F. Spiegelman, F. David (Eds.), Atomic Clusters and Nanoparticles, Les Houches Lectures Session LXXIII, Springer, Berlin, 2001, p. 221.

[16] P.W. Anderson, Phys. Rev. 110 (1958) 985.

[17] N.N. Bogolubov, Sov. Phys. JETP 34 (1958) 73.
[18] A. Vourdas, Phys. Rev. A 41 (1990) 1653.

[19] J. von Delft, A.D. Zaikin, D.S. Golubev, W. Tichy, Phys. Rev. Lett. 77 (1996) 3189.

[20] R.W. Richardson, Phys. Lett. 3 (1963) 277.

[21] A. Mastellone, G. Falci, R. Fazio, Phys. Rev. Lett. 80 (1998) 4542 .

[22] S.D. Berger, B.I. Halperin, Phys. Rev. B 58 (1998) 5213

[23] F. Braun, J. von Delft, Phys. Rev. Lett. 81 (1998) 4712.

[24] J. Dukelsky, G. Sierra, Phys. Rev. Lett. 83 (1999) 172.

[25] I.O. Kulik, H. Boyaci, Z. Gedik, Physica C 352 (2001) 46.

[26] K. Gottfried, Quantum Mechanics, Benjamin, New York, 1966, p. 320.

[27] J. von Delft, Ann. Phys. (Leipzig) 10 (2001) 3.

[28] B.R. Mottelson, The many-Body Problem, Ecole d'Eté de Physique Théorique Les Houches, Wiley, New York, 1959.

[29] Ö. Bozat, Z. Gedik, Solid State Commun. 120 (2001) 487. 